\title{
Automatic Granularity-Aware Parallelization of Programs with Predicates, Functions, and Constraints
}

\author{
Manuel Hermenegildo ${ }^{1,2}$ \\ http://www.cliplab.org/ herme \\ with Francisco Bueno, ${ }^{1}$ Manuel Carro, ${ }^{1}$ Amadeo Casas, ${ }^{2}$ \\ Pedro López, ${ }^{1}$ Edison Mera, ${ }^{1}$ and Jorge Navas ${ }^{2}$
}

Departments of Computer Science

${ }^{1}$ Technical University of Madrid, and

${ }^{2}$ University of New Mexico 


\section{Objectives}

- Parallelism (finally!) becoming mainstream thanks to multicore -even on laptops!

- Our objective herein is automatic parallelization of programs with predicates, functions, and constraints.

- We concentrate on detecting and-parallelism (corresponds to, e.g., loop parallelization, task parallelism, divide and conquer, etc.): 


\section{Objectives}

- Parallelism (finally!) becoming mainstream thanks to multicore -even on laptops!

- Our objective herein is automatic parallelization of programs with predicates, functions, and constraints.

- We concentrate on detecting and-parallelism (corresponds to, e.g., loop parallelization, task parallelism, divide and conquer, etc.):

\begin{tabular}{|c|c|}
\hline $\begin{aligned} \operatorname{fib}(0) & :=0 \\
\operatorname{fib}(1) & :=1 \\
\operatorname{fib}(N) & :=f i b(N-1)+f i b(N-2) \\
& :-N>1 .\end{aligned}$ & $\begin{array}{l}\operatorname{fib}(0,0) \\
\operatorname{fib}(1,1) . \\
\operatorname{fib}(N, F):- \\
N>1, \\
(N 1 \text { is } N-1, \\
\text { fib }(N 1, F 1)) \& \\
(N 2 \text { is } N-2, \\
\text { fib(N2, F2) }), \\
\text { F1+F2. }\end{array}$ \\
\hline
\end{tabular}

$\rightarrow$ Need to detect independent tasks. 
What is Independence? (for Functions, Predicates, Constraints, ...)

- Correctness: "same" solutions as sequential execution.

- Efficiency: execution time < than seq. program (or, at least, no-slowdown: $\leq$ ). (We assume parallel execution has no overhead in this first stage.)

- Running $s_{1} / / s_{2}$ :

\begin{tabular}{|l|c|c|c|}
\hline & Imperative & Functions & Constraints \\
\hline$s_{1}$ & $\mathrm{Y}:=\mathrm{W}+2 ;$ & $(+\mathrm{W} 2)$ & $\mathrm{Y}=\mathrm{W}+2$, \\
$s_{2}$ & $\mathrm{X}:=\mathrm{Y}+\mathrm{Z} ;$ & $(+\quad \mathrm{Z})$ & $\mathrm{X}=\mathrm{Y}+\mathrm{Z}$, \\
\hline & read-write deps & strictness & cost! \\
\hline
\end{tabular}




\section{What is Independence? (for Functions, Predicates, Constraints, ...)}

- Correctness: "same" solutions as sequential execution.

- Efficiency: execution time < than seq. program (or, at least, no-slowdown: $\leq$ ). (We assume parallel execution has no overhead in this first stage.)

- Running $s_{1} / / s_{2}$ :

\begin{tabular}{|l|c|c|c|}
\hline & Imperative & Functions & Constraints \\
\hline$s_{1}$ & $\mathrm{Y}:=\mathrm{W}+2 ;$ & $(+\mathrm{W} 2)$ & $\mathrm{Y}=\mathrm{W}+2$, \\
$s_{2}$ & $\mathrm{X}:=\mathrm{Y}+\mathrm{Z} ;$ & $(+\quad \mathrm{Z})$ & $\mathrm{X}=\mathrm{Y}+\mathrm{Z}$, \\
\hline & read-write deps & strictness & cost! \\
\hline
\end{tabular}

\begin{tabular}{|c|r|}
\hline \multicolumn{2}{|c|}{ For Predicates (multiple procedure definitions): } \\
\hline main:- & $\mathrm{p}(\mathrm{X}):-\mathrm{X}=\mathrm{a}$. \\
$s_{1} \quad \mathrm{p}(\mathrm{X})$, & $\mathrm{q}(\mathrm{X}):-\mathrm{X}=\mathrm{b}$, large computation. \\
$s_{2} \mathrm{q}(\mathrm{X})$, & $\mathrm{q}(\mathrm{X}):-\mathrm{X}=\mathrm{a}$. \\
& \\
& \\
\hline Agrite $(\mathrm{X})$. & \\
\hline
\end{tabular}

- Independence: condition that guarantees correctness and efficiency. 


\section{Independence}

- Strict independence (suff. condition): no "pointers" shared at run-time:

- Non-strict independence: only one thread accesses each shared variable.

- Requires global analysis.

- Required in programs using "incomplete structures" (difference lists, etc.). 


\section{Independence}

- Strict independence (suff. condition): no "pointers" shared at run-time:

- Non-strict independence: only one thread accesses each shared variable.

- Requires global analysis.

- Required in programs using "incomplete structures" (difference lists, etc.).

- Constraint independennce -more involved:

$$
\begin{aligned}
& \text { main :- } \mathrm{X} .>. \mathrm{Y}, \mathrm{Z} .>. \mathrm{Y}, \mathrm{p}(\mathrm{X}) \& \mathrm{q}(\mathrm{Z}), \ldots \\
& \text { main :- } \mathrm{X} .>\text {. Y, Y .>. } \mathrm{Z}, \mathrm{p}(\mathrm{X}) \& \mathrm{q}(\mathrm{Z}), \ldots
\end{aligned}
$$




\section{Independence}

- Strict independence (suff. condition): no "pointers" shared at run-time:

- Non-strict independence: only one thread accesses each shared variable.

- Requires global analysis.

- Required in programs using "incomplete structures" (difference lists, etc.).

- Constraint independennce -more involved:

$$
\begin{aligned}
& \text { main :- } \mathrm{X} .>. \mathrm{Y}, \mathrm{Z} .>. \mathrm{Y}, \mathrm{p}(\mathrm{X}) \& \mathrm{q}(\mathrm{Z}), \ldots \\
& \text { main :- } \mathrm{X} .>. \mathrm{Y}, \mathrm{Y} .>. \mathrm{Z}, \mathrm{p}(\mathrm{X}) \& \mathrm{q}(\mathrm{Z}), \ldots
\end{aligned}
$$

Sufficient a-priori condition: given $g_{1}(\bar{x})$ and $g_{2}(\bar{y}), c$ state just before them:

$$
(\bar{x} \cap \bar{y} \subseteq \operatorname{def}(c)) \text { and }\left(\exists_{-\bar{x}} c \wedge \exists_{-\bar{y}} c \rightarrow \exists_{-\bar{y}\lrcorner \bar{x}} c\right)
$$

$($ def $(c)=$ set of variables constrained to a unique value in $c)$

- For $c=\{x>y, z>y\} \quad \bar{\exists}_{-\{x\}} c=\bar{\exists}_{-\{z\}} c=\bar{\exists}_{-\{x, z\}} c=$ true

- For $c=\{x>y, y>z\} \quad \bar{\exists}_{-\{x\}} c=\bar{\exists}_{-\{z\}} c=$ true,$\quad \bar{\exists}_{\{x, z\}} c=x>z$

Approximation: presence of "links" through the store. 


\section{Parallelization Process}

- Conditional dependency graph (of some code segment, e.g., a clause):

- Vertices: possible tasks (statements, calls,...),

- Edges: possible dependencies (labels: conditions needed for independence).

- Local or global analysis used to reduce/remove checks in the edges.

- Annotation process converts graph back to parallel expressions in source.

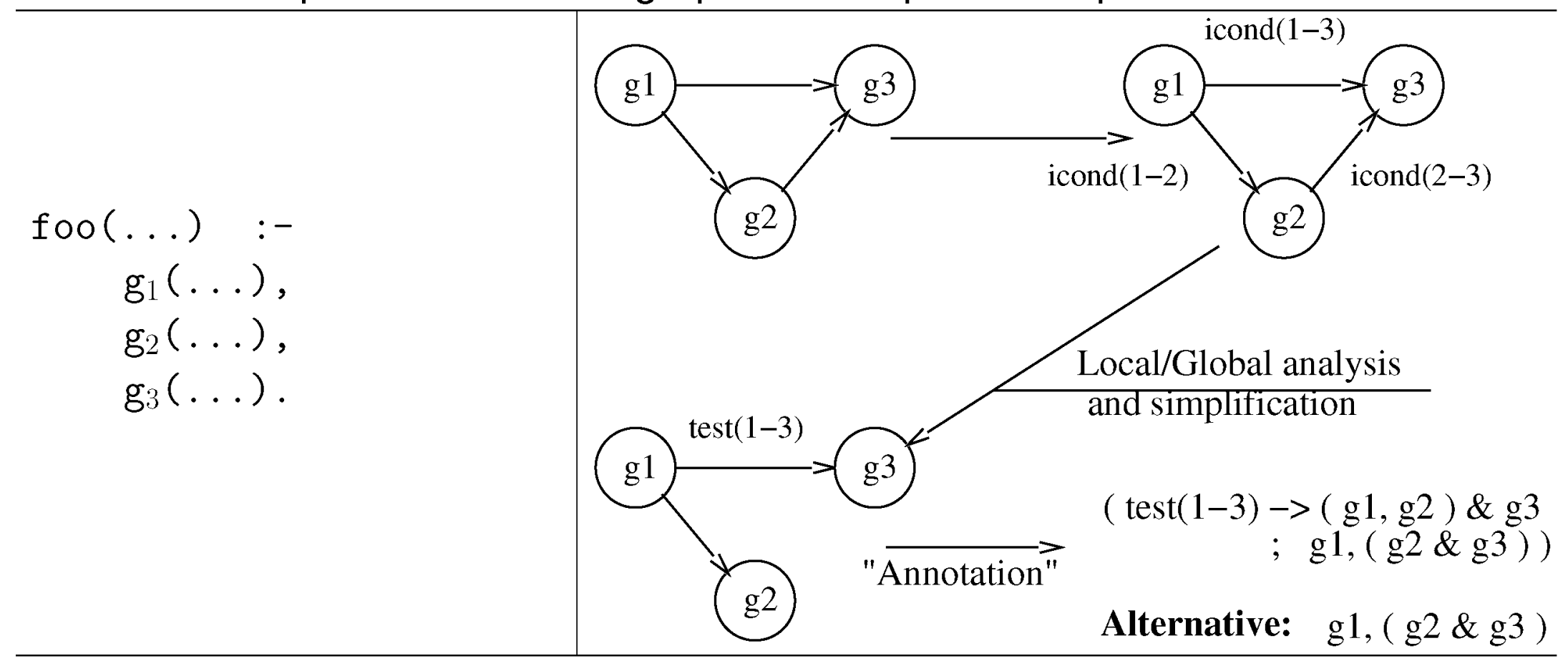




\section{Concrete System Used in Examples: Ciao}

- One of the popular Prolog/CLP systems (supports ISO-Prolog fully).

- At the same time, new-generation multi-paradigm language/prog.env. with:

- Predicates, constraints, functions (including lazyness), higher-order, ... (And Prolog impure features only present as compatibility libraries.) 


\section{Concrete System Used in Examples: Ciao}

- One of the popular Prolog/CLP systems (supports ISO-Prolog fully).

- At the same time, new-generation multi-paradigm language/prog.env. with:

- Predicates, constraints, functions (including lazyness), higher-order, ... (And Prolog impure features only present as compatibility libraries.)

- Assertion language for expressing rich program properties

(types, shapes, pointer aliasing, non-failure, determinacy, termination, data sizes, cost, ...).

- Static debugging, verification, program certification, PCC, ... 


\section{Concrete System Used in Examples: Ciao}

- One of the popular Prolog/CLP systems (supports ISO-Prolog fully).

- At the same time, new-generation multi-paradigm language/prog.env. with:

- Predicates, constraints, functions (including lazyness), higher-order, ...

(And Prolog impure features only present as compatibility libraries.)

- Assertion language for expressing rich program properties

(types, shapes, pointer aliasing, non-failure, determinacy, termination, data sizes, cost, ...).

- Static debugging, verification, program certification, PCC, ...

- Parallel, concurrent, and distributed execution primitives.

- Automatic parallelization.

- Automatic granularity and resource control. 


\section{Concrete System Used in Examples: Ciao}

- One of the popular Prolog/CLP systems (supports ISO-Prolog fully).

- At the same time, new-generation multi-paradigm language/prog.env. with:

- Predicates, constraints, functions (including lazyness), higher-order, ...

(And Prolog impure features only present as compatibility libraries.)

- Assertion language for expressing rich program properties

(types, shapes, pointer aliasing, non-failure, determinacy, termination, data sizes, cost, ...).

- Static debugging, verification, program certification, PCC, ...

- Parallel, concurrent, and distributed execution primitives.

- Automatic parallelization.

- Automatic granularity and resource control.

- + several control rules (e.g., bf, id, Andorra), objects, syntactic/semantic extensibility, LGPL, ... 


\section{Some Speedups (for different analysis abstract domains)}

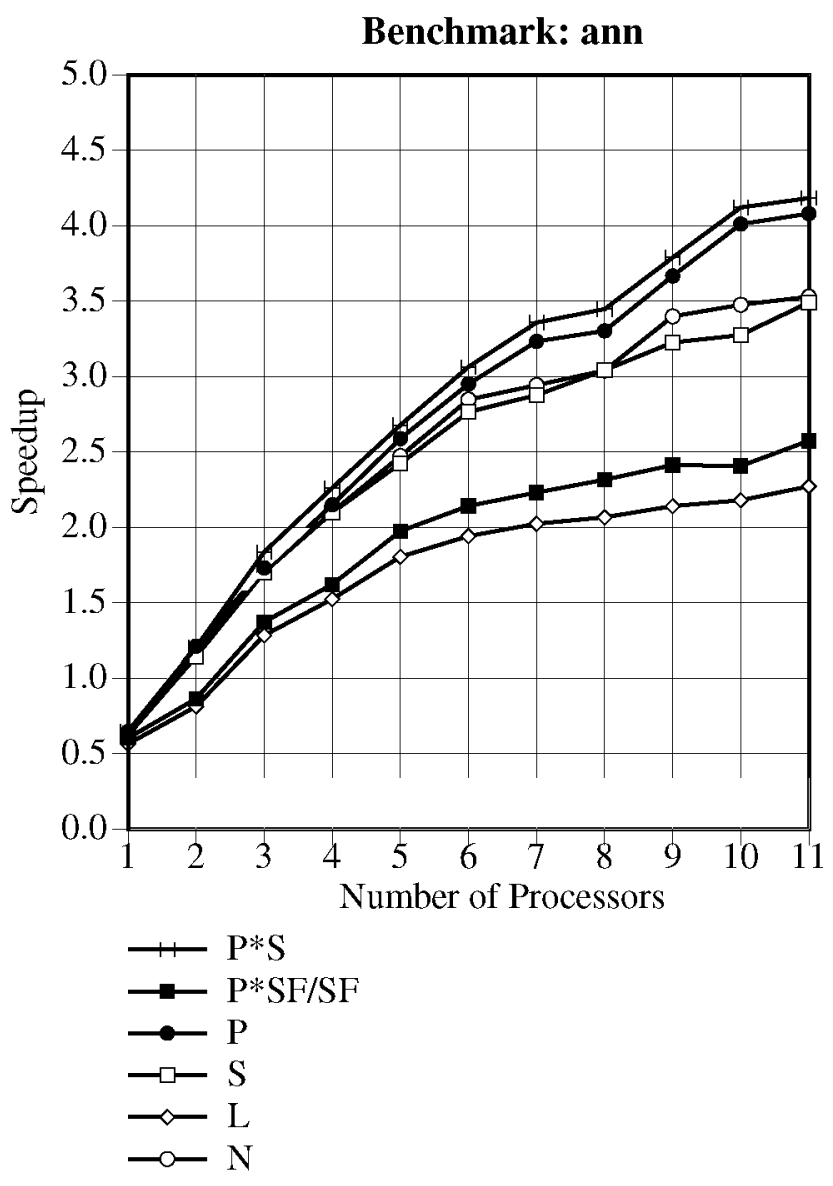

The parallelizer, self-parallelized 


\section{Granularity Control}

- Replace parallel with sequential execution based on task size and overheads.

- Cannot be done completely at compile-time: cost often depends on input (hard to approximate at compile time, even w/abstract interpretation).

main :- $\operatorname{read}(X), \operatorname{read}(Z)$, inc_all $_{(X, Y)} \& \mathrm{r}(\mathrm{Z}, \mathrm{M}), \ldots$ 


\section{Granularity Control}

- Replace parallel with sequential execution based on task size and overheads.

- Cannot be done completely at compile-time: cost often depends on input (hard to approximate at compile time, even w/abstract interpretation). main :- $\operatorname{read}(X), \operatorname{read}(Z)$, inc_all $_{(X, Y)} \& \mathrm{r}(\mathrm{Z}, \mathrm{M}), \ldots$

- Our approach:

- Derive at compile-time cost functions (to be evaluated at run-time) that efficiently bound task size (lower, upper bounds).

- Transform programs to carry out run-time granularity control.

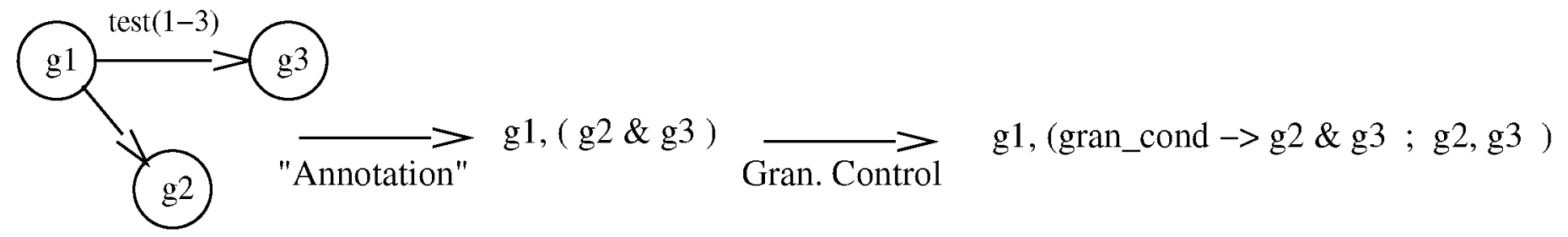

- For inc_all, (assuming "threshold" is 100 units):

$$
\begin{aligned}
& \text { main }:-\operatorname{read}(\mathrm{X}), \operatorname{read}(\mathrm{Z}),(2 * \text { length }(\mathrm{X})+1>100->\text { inc_all }(\mathrm{X}, \mathrm{Y}) \& \mathrm{r}(\mathrm{Z}, \mathrm{M}) \\
& \text {; inc_all }(X, Y), r(Z, M)) \text {, }
\end{aligned}
$$




\section{Inference of Bounds on Argument Sizes and Procedure Cost in CiaoPP}

1. Perform type/mode inference:

$$
\text { :- true inc_all }(\mathrm{X}, \mathrm{Y}) \text { : list (X, int), } \operatorname{var}(\mathrm{Y}) \Rightarrow \text { list (Y,int). }
$$

2. Infer size measures: list length.

3. Use data dependency graphs to determine the relative sizes of structures that variables point to at different program points - infer argument size relations:

$\operatorname{Size}_{\text {inc_all }}^{2}(0)=0$ (boundary condition from base case),

$\operatorname{Size}_{\text {inc_all }}^{2}(n)=1+\operatorname{Size}_{\text {inc_all }}^{2}(n-1)$.

Sol $=\operatorname{Size}_{\text {inc_all }}^{2}(n)=n$.

4. Use this, set up recurrence equations for the computational cost of procedures:

$\operatorname{Cost}_{\text {inc_all }}^{\mathrm{L}}(0)=1$ (boundary condition from base case),

$\operatorname{Cost}_{\text {inc_all }}^{\mathrm{L}}(n)=2+\operatorname{Cost}_{\text {inc_all }}^{\mathrm{L}}(n-1)$.

Sol $=\operatorname{Cost}_{\text {inc_all }}^{\mathrm{L}}(n)=2 n+1$.

- We obtain lower/upper bounds on task granularities.

- Non-failure (absence of exceptions) analysis needed for lower bounds. 


\section{Refinements (1): Granularity Control Optimizations}

- Simplification of cost functions:

$\ldots$... ( length $(X)>50 \rightarrow$ inc_all $(X, Y)$ \& $r(Z, M)$

; inc_all $(X, Y), r(Z, M)), \ldots$ 


\section{Refinements (1): Granularity Control Optimizations}

- Simplification of cost functions:

$\ldots$, ( length $(X)>50 \rightarrow$ inc_all $(X, Y)$ \& $r(Z, M)$

; inc_all $(X, Y), r(Z, M)), \ldots$

$\ldots$, ( length_gt $(\mathrm{LX}, 50) \rightarrow$ inc_all $(X, Y)$ \& $r(Z, M)$

; inc_all $(X, Y), r(Z, M)), \ldots$ 


\section{Refinements (1): Granularity Control Optimizations}

- Simplification of cost functions:

$\ldots$, ( length $(X)>50 \rightarrow$ inc_all $(X, Y)$ \& $r(Z, M)$

; inc_all $(X, Y), r(Z, M)), \ldots$

$\ldots$ ( length_gt $(\mathrm{LX}, 50) \rightarrow$ inc_all $(X, Y)$ \& $r(Z, M)$

; inc_all $(X, Y), r(Z, M)), \ldots$

- Complex thresholds: use also communication cost functions, load, ... Example: Assume CommCost $\left(i n c \_a l l(X)\right)=0.1($ length $(X)+$ length $(Y))$. We know $u b$ length $(Y)$ (actually, exact size) = length $(X)$; thus:

$$
\begin{array}{r}
2 \text { length }(X)+1>0.1(\text { length }(X)+\text { length }(X)) \cong \\
2 \text { length }(X)>0.2 \text { length }(X) \equiv
\end{array}
$$

$$
2>0.2
$$




\section{Refinements (1): Granularity Control Optimizations}

- Simplification of cost functions:

$$
\begin{aligned}
& \ldots, \text { ( length }(X)>50 \rightarrow \operatorname{inc}_{-} a l l(X, Y) \text { \& } r(Z, M) \\
& \text {; inc_all }(X, Y), r(Z, M)), \ldots \\
& \text {..., ( length_gt }(\mathrm{LX}, 50) \rightarrow \text { inc_all }(X, Y) \text { \& } r(Z, M) \\
& \text {; inc_all }(X, Y), r(Z, M)), \ldots
\end{aligned}
$$

- Complex thresholds: use also communication cost functions, load, ... Example: Assume CommCost $\left(i n c \_a l l(X)\right)=0.1($ length $(X)+$ length $(Y))$. We know $u b$ length $(Y)$ (actually, exact size) $=$ length $(X)$; thus:

$$
\begin{array}{r}
2 \text { length }(X)+1>0.1(\text { length }(X)+\text { length }(X)) \cong \\
2 \text { length }(X)>0.2 \text { length }(X) \equiv
\end{array}
$$

Guaranteed speedup for any data size!

$$
\Leftarrow \quad 2>0.2
$$




\section{Refinements (1): Granularity Control Optimizations}

- Simplification of cost functions:

$$
\begin{aligned}
& \ldots \text { ( length }(X)>50 \rightarrow \text { inc_all }(X, Y) \& r(Z, M) \\
& \text {; inc_all }(X, Y), r(Z, M)), \ldots \\
& \text {..., ( length_gt }(\mathrm{LX}, 50) \rightarrow \text { inc_all }(X, Y) \& r(Z, M) \\
& \text {; inc_all }(X, Y), r(Z, M)), \ldots
\end{aligned}
$$

- Complex thresholds: use also communication cost functions, load, ... Example: Assume CommCost $($ inc_all $(X))=0.1($ length $(X)+$ length $(Y))$. We know $u b$ length $(Y)$ (actually, exact size) $=$ length $(X)$; thus:

$$
\begin{array}{r}
2 \text { length }(X)+1>0.1(\text { length }(X)+\text { length }(X)) \cong \\
2 \text { length }(X)>0.2 \text { length }(X) \equiv
\end{array}
$$

Guaranteed speedup for any data size! $\Leftarrow \quad 2>0.2$

- Checking of data sizes can be stopped once under threshold.

- Data size computations can often be done on-the-fly.

- Static task clustering (loop unrolling), static placement, etc. 


\section{Granularity Control System Output Example}

g_qsort ( [], []).

g_qsort([First|L1], L2) :-

partition3o4o(First, L1, Ls, Lg, Size_Ls, Size_Lg),

Size_Ls $>20 \rightarrow$ (Size_Lg $>20 \rightarrow$ g_qsort(Ls, Ls2) \& g_qsort(Lg, Lg2)

; g_qsort (Ls, Ls2) , s_qsort (Lg, Lg2))

; $\quad($ Size_Lg $>20 \rightarrow$ s_qsort(Ls, Ls2) , g_qsort (Lg, Lg2)

; s_qsort (Ls, Ls2), s_qsort (Lg, Lg2))),

append(Ls2, [First|Lg2], L2).

partition $3040(\mathrm{~F},[],[],[], 0,0)$.

partition3o4o(F, $[\mathrm{X} \mid \mathrm{Y}],[\mathrm{X} \mid \mathrm{Y} 1], \mathrm{Y} 2, \mathrm{SL}, \mathrm{SG})$ :-

$\mathrm{X}=<\mathrm{F}, \operatorname{partition} 3040(\mathrm{~F}, \mathrm{Y}, \mathrm{Y1}, \mathrm{Y} 2, \mathrm{SL1}, \mathrm{SG}), \mathrm{SL}$ is $\mathrm{SL1}+1$. partition3o4o(F, $[X \mid Y], Y 1,[X \mid Y 2], S L, S G):-$

$\mathrm{X}>\mathrm{F}, \operatorname{partition3040}(\mathrm{F}, \mathrm{Y}, \mathrm{Y} 1, \mathrm{Y} 2, \mathrm{SL}, \mathrm{SG} 1), \mathrm{SG}$ is $\mathrm{SG1}+1$. 


\section{Refinements (2): Granularity-Aware Annotation}

- With classic annotators (MEL, UDG, CDG, ...) we applied granularity control after parallelization:

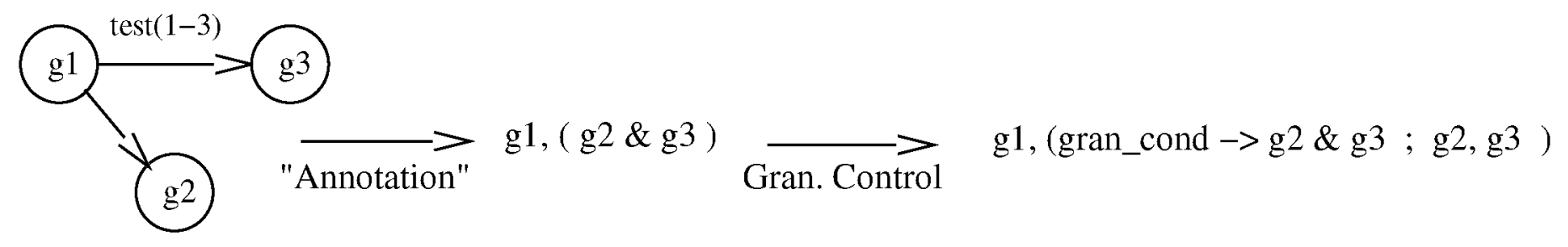




\section{Refinements (2): Granularity-Aware Annotation}

- With classic annotators (MEL, UDG, CDG, ...) we applied granularity control after parallelization:

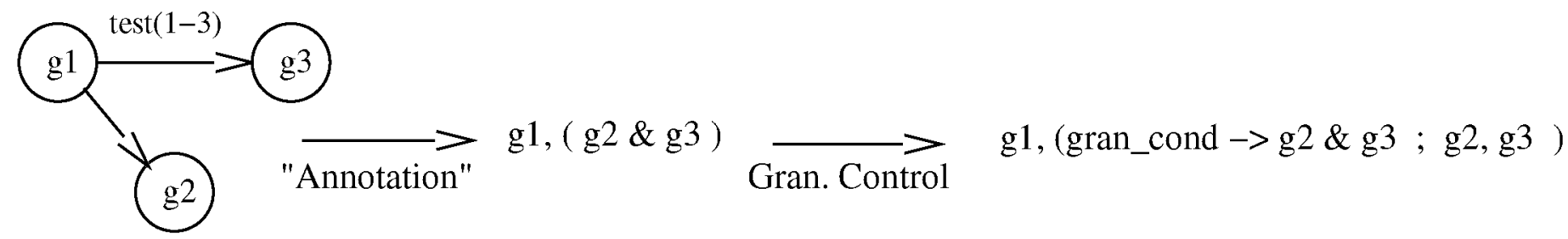

- Developed new annotation algorithm that takes task granularity into account:

- Annotation is a heuristic process (several alternatives possible).

- Taking task granularity into account during annotation can help make better choices and speed up annotation process.

- Tasks with larger cost bounds given priority, small ones not parallelized.

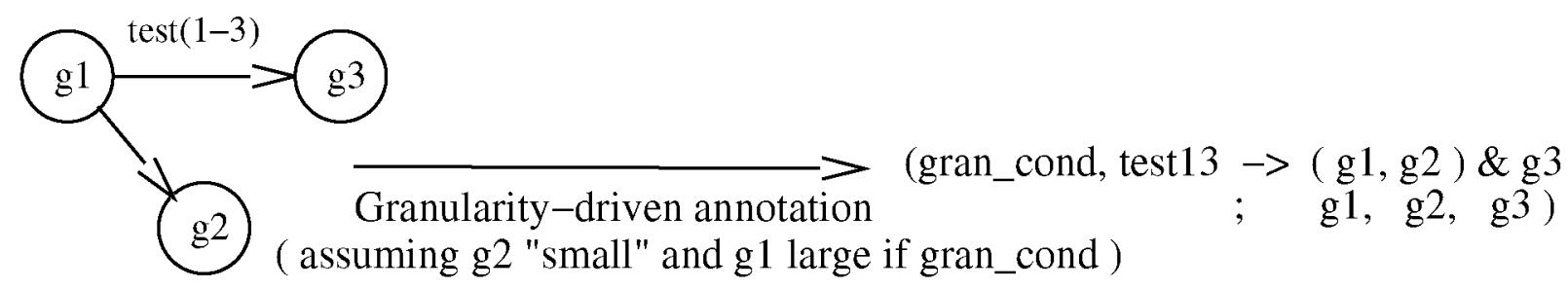




\section{Granularity-Aware Annotation: Concrete Example}

- Consider the clause: $p:-a, b, c, d, e$.

- Assume that the dependencies detected between the subgoals of $\mathrm{p}$ are given by:

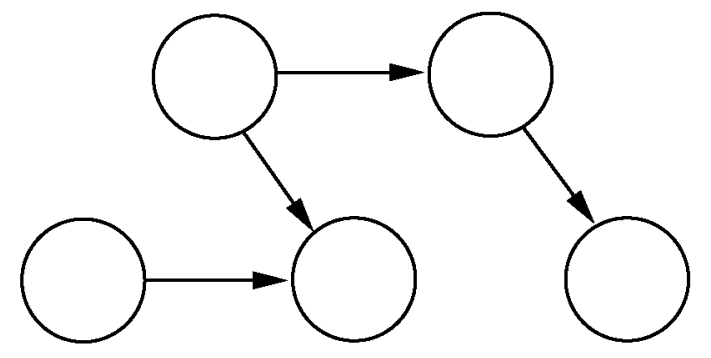

- Assume also that:

$$
T(a)<T(c)<T(e)<T(b)<T(d),
$$

where $T(i)<T(j)$ means: cost of subgoal $i$ is smaller than the cost of $j$. 


\section{Granularity-Aware Annotation: Concrete Example}

- Consider the clause: $p:-a, b, c, d, e$.

- Assume that the dependencies detected between the subgoals of $p$ are given by:

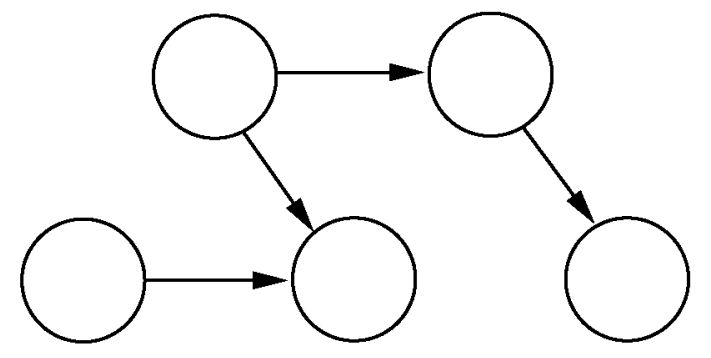

- Assume also that:

$$
T(a)<T(c)<T(e)<T(b)<T(d),
$$

where $T(i)<T(j)$ means: cost of subgoal $i$ is smaller than the cost of $j$.

$$
\left|\begin{array}{ll}
\text { MEL annotator: } & (\mathrm{a}, \mathrm{b} \& \mathrm{c}, \mathrm{d} \& \mathrm{e}) \\
\text { UDG annotator: } & (\mathrm{c} \&(\mathrm{a}, \mathrm{b}, \mathrm{e}), \mathrm{d}) \\
\text { Granularity-aware: } & (\mathrm{a}, \mathrm{c},(\mathrm{b} \& \mathrm{~d}), \mathrm{e})
\end{array}\right|
$$




\section{Refinements (3): Using Execution Time Bounds/Estimates}

- Use estimations/bounds on execution time for controlling granularity (instead of steps/reductions).

- Execution time generally dependent on platform characteristics ( $\approx$ constants) and input data sizes (unknowns).

- Platform-dependent, one-time calibration using fixed set of programs:

- Obtains value of the platform-dependent constants (costs of basic operations).

- Platform-independent, compile-time analysis:

- Infers cost functions (using modification of previous method), which return count of basic operations given input data sizes.

- Incorporate the constants from the calibration.

$\rightarrow$ we obtain functions yielding execution times depending on size of input.

- Predicts execution times with reasonable accuracy (challenging!).

- Improving by taking into account lower level factors (current work). 


\section{Execution Time Estimation: Concrete Example}

- Consider nrev with mode:

:- pred nrev/2 : list(int) * var.

- Estimation of execution time for a concrete input - consider:

$A=[1,2,3,4,5], \bar{n}=$ length $(A)=5$

\begin{tabular}{|c|r|c|r|r|}
\hline & Once & Static Analysis & \multicolumn{2}{|c|}{ Application } \\
\hline component & $K_{\omega_{i}}$ & Cost $_{\mathrm{p}}\left(I\left(\omega_{i}\right), \bar{n}\right)=C_{i}(\bar{n})$ & $C_{i}(5)$ & $K_{\omega_{i}} \times C_{i}(5)$ \\
\hline step & 21.27 & $0.5 \times n^{2}+1.5 \times n+1$ & 21 & 446.7 \\
nargs & 9.96 & $1.5 \times n^{2}+3.5 \times n+2$ & 57 & 567.7 \\
giunif & 10.30 & $0.5 \times n^{2}+3.5 \times n+1$ & 31 & 319.3 \\
gounif & 8.23 & $0.5 \times n^{2}+0.5 \times n+1$ & 16 & 131.7 \\
viunif & 6.46 & $1.5 \times n^{2}+1.5 \times n+1$ & 45 & 290.7 \\
vounif & 5.69 & $n^{2}+n$ & 30 & 170.7 \\
\hline Execution time $\bar{K}_{\Omega} \bullet \overline{\text { Cost }}_{\mathrm{p}}(\overline{I(\Omega)}, \bar{n}):$ & & 1926.8 \\
\hline
\end{tabular}




\section{Visualization of And-parallelism - (small) qsort, 4 processors}

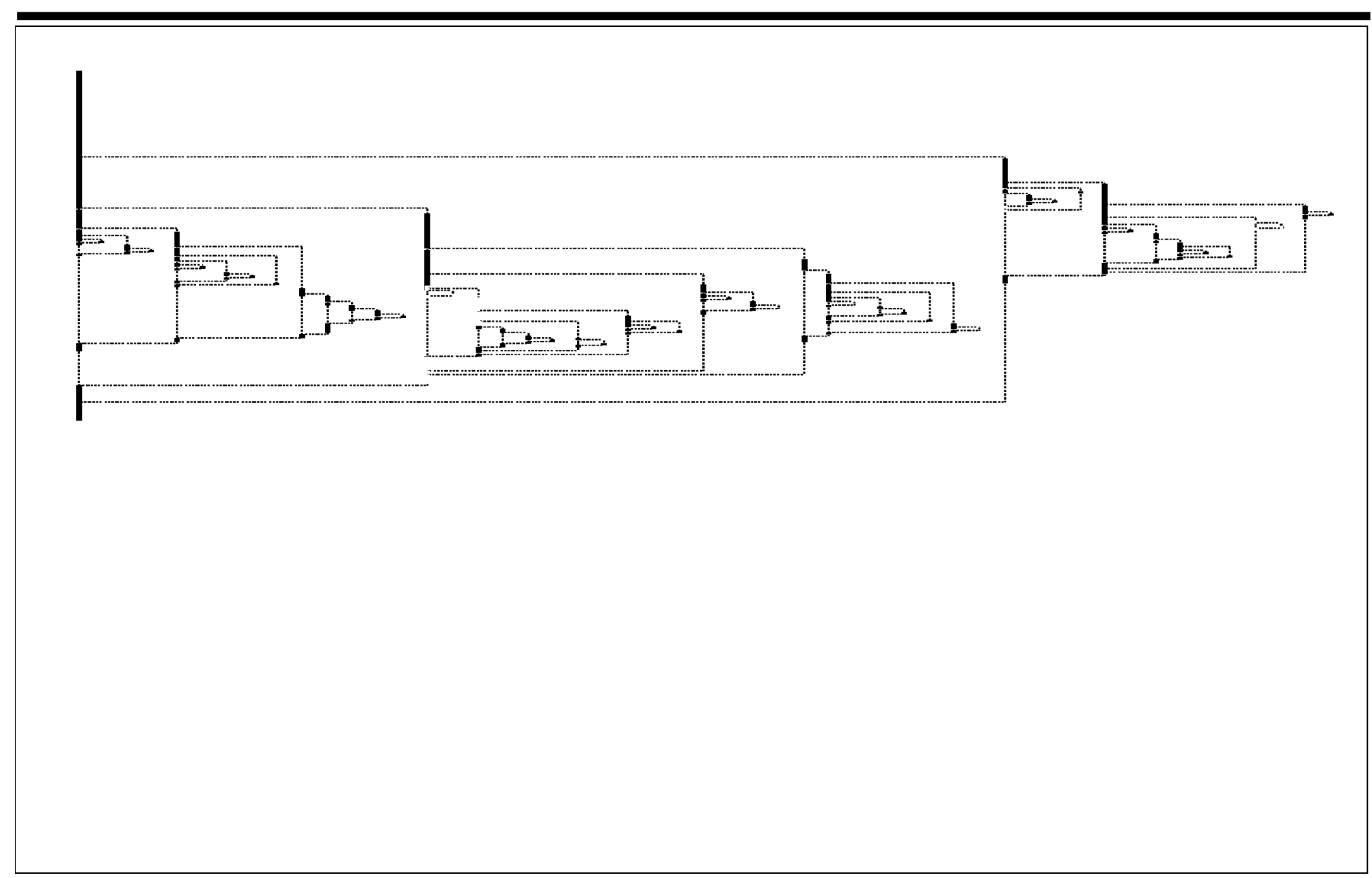


Fib 15, 1 processor

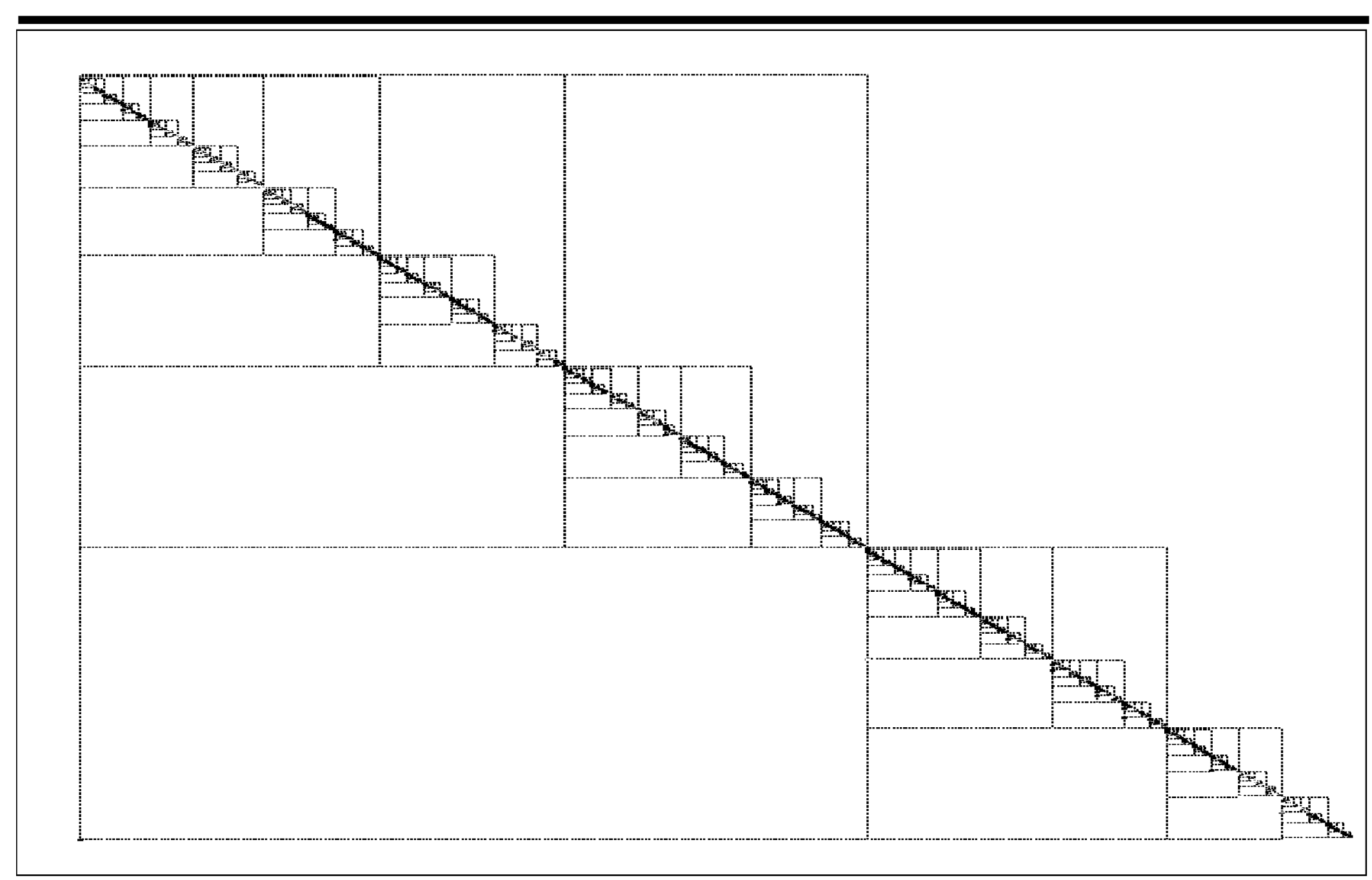


Fib 15, 8 processors (same scale)

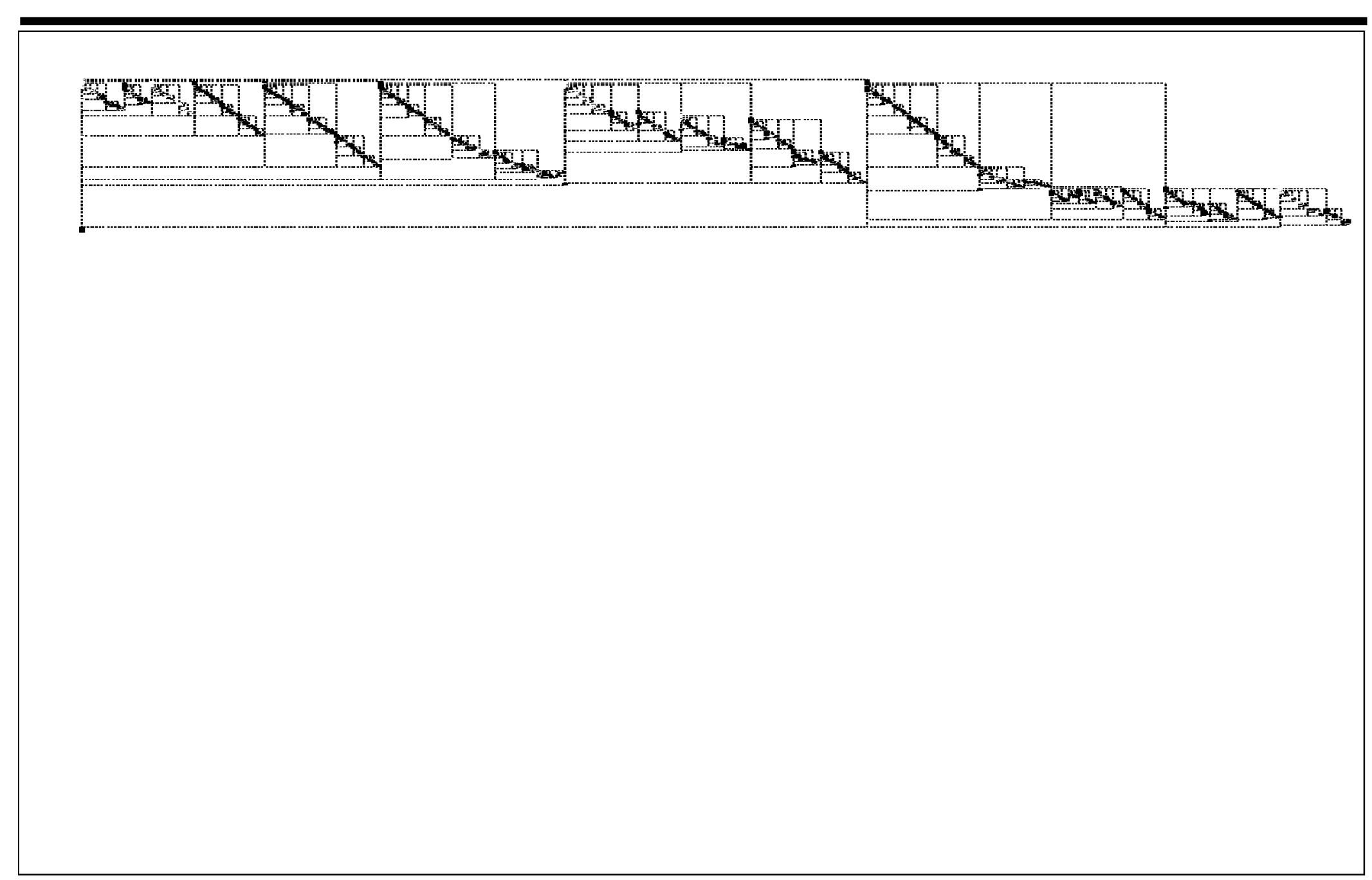


Fib 15, 8 processors (full scale)

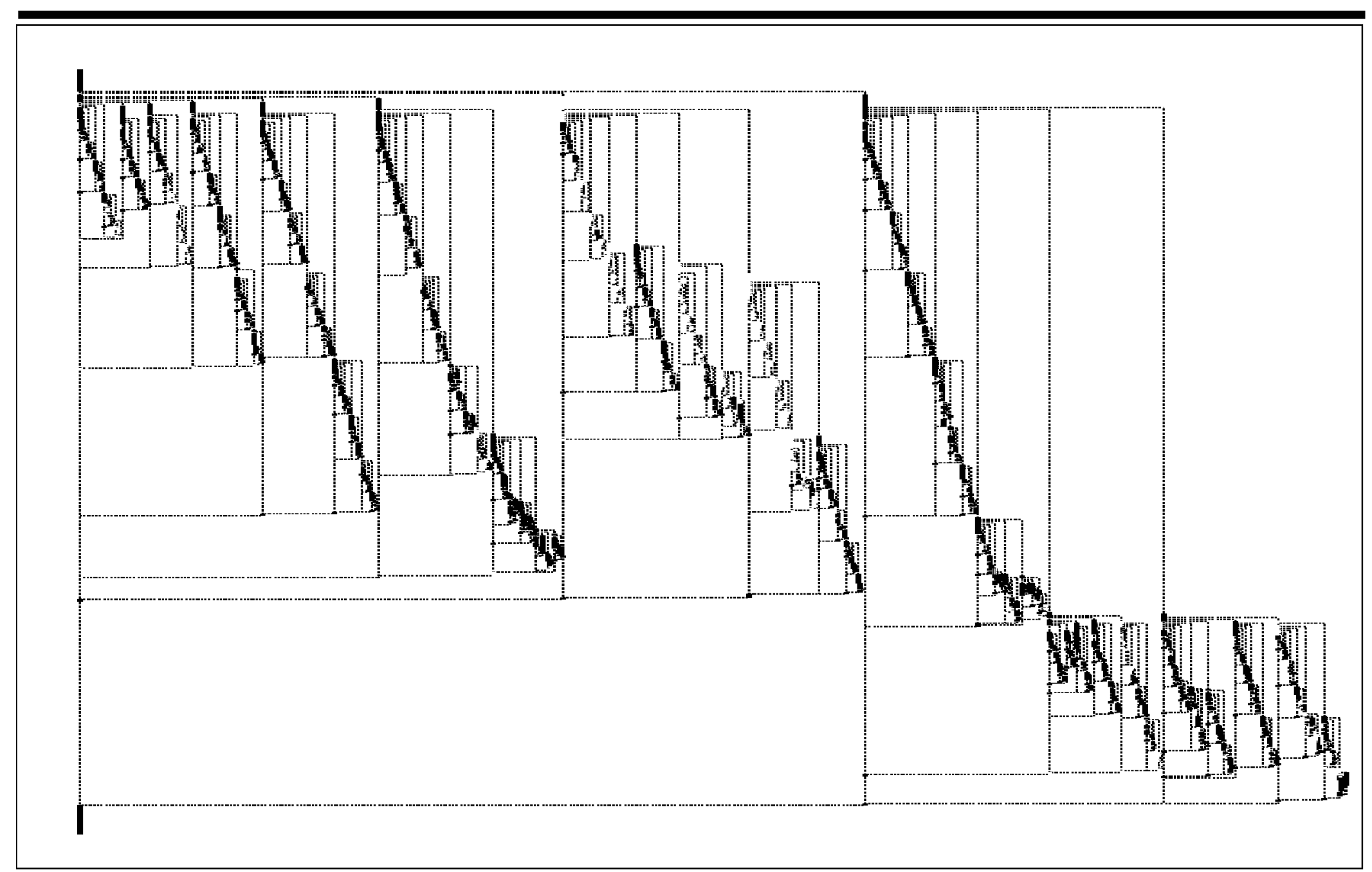


Fib 15, 8 processors, with granularity control (same scale)

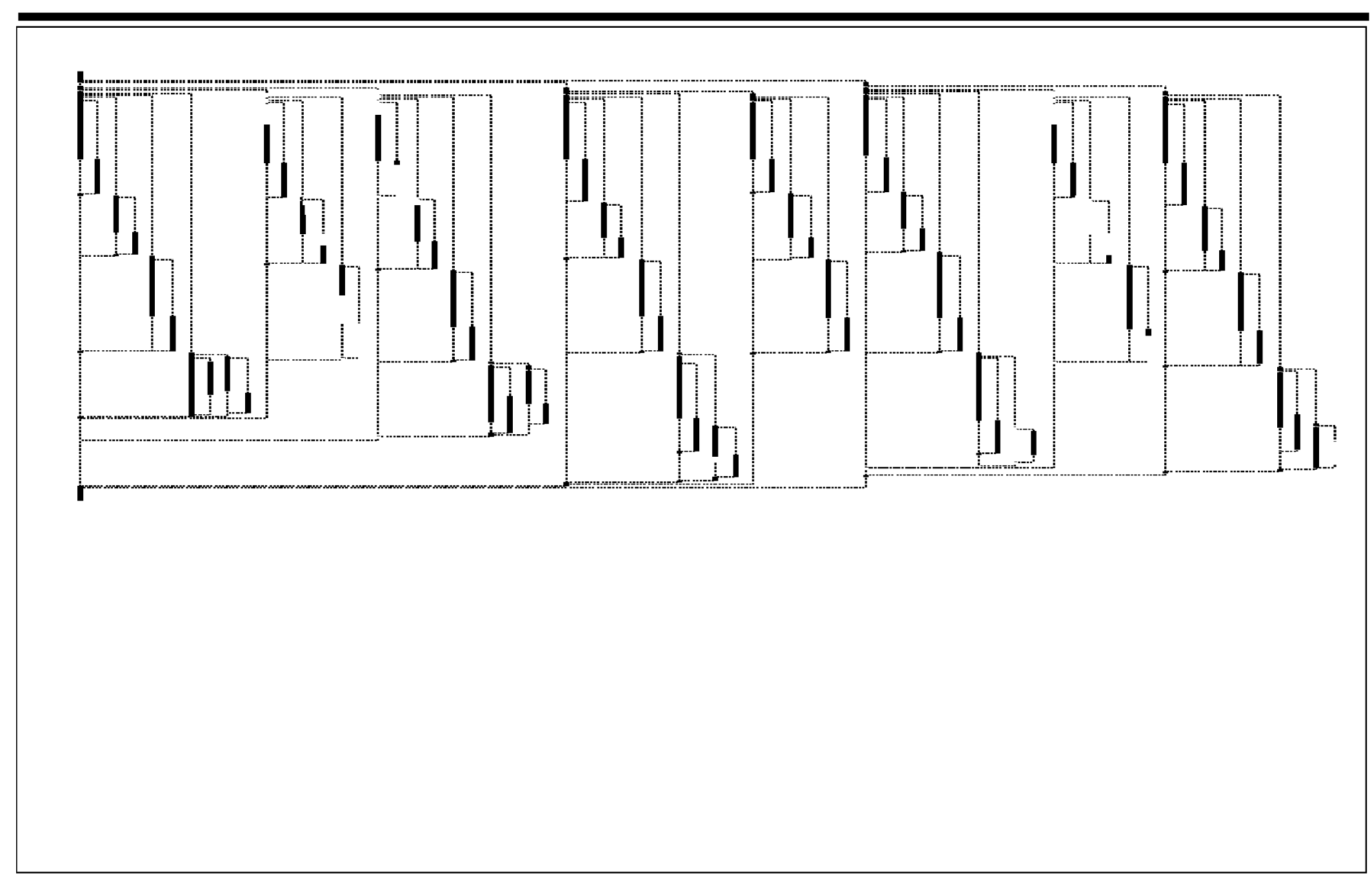

